\title{
Cosmology with variable $G$ and nonlinear electrodynamics
}

\author{
Gabriel W. Joseph ${ }^{1, *}$ and Ali Övgün ${ }^{1, \dagger}$ \\ ${ }^{1}$ Physics Department, Faculty of Arts and Sciences, Eastern Mediterranean University, Famagusta, North Cyprus, via Mersin 10, Turkey
}

(Dated: July 7, 2020)

\begin{abstract}
In a bid to resolve lingering problems in cosmology, more focus is being tilted towards cosmological models in which physical constants of nature are not necessarily real constants, but varying with cosmic time. In this paper we have study cosmology in nonlinear electrodynamics with the Newton's gravitational constant $G$ not a constant but vary in form of power-law of the scale factor of the universe. The evolution of the scale factor $a(t)$ is studied in this model which depends on nonlinear electrodynamics fine tuning term of $\alpha$. Then we check the stability of the model using the speed of sound.
\end{abstract}

PACS numbers: 95.36.+x, 98.80.-k

Keywords: Cosmology, nonlinear electrodynamics, inflation, acceleration of the universe, causality, classical stability, variable $G$

\section{INTRODUCTION}

Remarkable achievements gained in the field of Cosmology over the last decades can be attributed to observations from Cosmic Microwave Background $(\mathrm{CMB})$ radiation and type-Ia supernovae suggesting that cosmic expansion is accelerating [1]. Making used of Maxwell's electrodynamics as source, with the Friedmann-Robertson-Walker spacetime, which is the pivot of the Standard Cosmological Model (SCM) leads to an initial singularity - breakdown in the geometrical structure of space and time at a finite time in the past. This initial singularity around the very early universe has revealed an unpredictable large value of the energy density and curvature of spacetime, thus departing from the domain of the application of the SCM. This setback has also given rise to other secondary problems in cosmology such as the horizon problem (as the universe approaches its causally correlated region, it appears to be too homogeneous at large scales), flatness problem and monopole problem. The secondary problems have been resolved by using geometric scalar fields. Magnetic universe models which portray no initial singularity due to strong electromagnetic field in the modified Nonlinear Electrodynamics (NED) in the early universe has been used in the literature to resolve this puzzle [2-18].

By introducing cosmological constant $\Lambda$, or scalar field with a type of potential function or modifying the theory of gravity with the aid of some function, $f(r)$ one can effectively explain the acceleration of the universe [19]. All these models are not without their setbacks, for instance; the smallness of the cosmological constant when compared with the vacuum energy is difficult to explain. Furthermore, the choice of $f(r)$ function in the modified gravity is not unique whereas, in scalar-tensor theory, much potential leads to cosmic inflation and acceleration of the universe. When NED is coupled to a

\footnotetext{
* gabrielwjoseph@gmail.com

† ali.ovgun@emu.edu.tr; https:/ / www.aovgun.com
}

gravity field, may give the necessary negative pressure and enhance cosmic inflation. Here, it is interesting to explore a new model of NED with variable gravitational constant and stochastic magnetic field drives inflation of the universe.

Magnetic fields at different levels in the past have effects on various cosmological and astrophysical phenomena observed in the universe. Frozen in ionized baryon are magnetic fields whose strength is of the order of $1.0 \mu G$ on larger scale compared to that of galactic clusters corresponding to Primordial Magnetic Fields (PMF). Adequate knowledge of the PMF offers information about the early universe between inflationary and recombination era. Such magnetic fields originate from cosmic phase transition with bluer spectrum and vector potential produced at inflation era. Stochastic inconsistencies of EM field in relativistic electron-positron plasma produce PMF from thermal instabilities in the pre-recombination plasma. The plasma sustains the magnetic fluctuations in the early universe before the era of Big Bang nucleosynthesis during the radiation dominated era.

Using the new type of NED containing a stochastic magnetic background with nonvanishing $B^{2}$ gives cosmological models void of initial singularities. Other cosmological models without premordial singularity such as non-minimal coupling, Varying Speed of Light (VSL), quantum gravity effect, Lagrange with quadratic term, inflation by scalar fields, NED without modification of general relativity have been introduced in the literature to solve the puzzle of cosmology and mystery of inflation [19-40].

Max Born and Leopold Infeld used the idea of Gustav who in 1909 attempted to construct a pure electromagnetic theory of charge particles to proposed a new theory in 1934, fully relativistic and gauge invariant nonlinear electrodynamics [41]. Born-Infeld proposed a nonlinear Lagrange with an interesting attribute of transforming to Maxwell's theory for low electromagnetic fields. Since there are no new degrees of freedom such as scalar fields or branes, works of cosmology described 
by NED should have some interesting features of cosmic importance. The sources of cosmic inflation can be trace to nonlinear electromagnetic radiation which is explained by modified Maxwell's equations. When coupled with gravitational field, NED may give negative pressure and also can lead to cosmic inflation [19]. The evolution of the universe when explored with a new NED model such that electromagnetic field coupled to gravitational field prevents cosmic singularity at the big bang. The electromagnetic and gravitational fields were very strong during the evolution of the early universe, thereby leading to quantum correction and giving birth to NED [23-25]. One can present a model of the universe filled with Born-Infeld type of nonlinear electromagnetic field which is inhomogeneous and anisotropic without singularity.

In recent times, interest is being geared towards cosmological models in which physical constants of nature are varying with time [42-44]. For instance, in the VSL theory, most pending problems of standard cosmological models are being resolved without considering inflation [45-47]. The Newtonian gravitational constant $G$, occurs in the source term of Einstein's field equation of general theory of relativity; which is a fundamental equation for developing every models of cosmology. In the Einstein's field equation, $G$ acts as a coupling constant between geometry of spacetime and matter. In quantum mechanics, $\mathrm{G}$ is essential in the definition of Planck constant [48]. While in SCM, $G$ is an invariant quantity, it has been noted that there are significant evidence that gravitational constant $G$ can be varying in a time [49]. Spurred by the discovery huge inconsistency between distance scales in force field, Dirac had proposed the time dependent cosmological model. In other to unify gravitation and elementary particle Physics, Einstein's theory with time varying $G$ is already in the literature [46, 50-53, 55-61]. Singh [53] has presented a cosmological model with $G \sim a^{2 / \alpha}$, where $a$ is the scale factor of the universe and $\alpha$ a constant. When either $G$ or the cosmological constant $\Lambda$ is varied, the Einstein field equation is still preserved. It has been shown that, variable Newtonian gravitation constant can account for dark energy and most of its effects, and current dynamical dark energy models using time-dependent cosmological constant terms are being considered [54].

In this paper, we discussed a model of variable $G$ while still maintaining the conservation law of energy momentum tensor with $G \propto a^{m}$. We show that variable $G$ in the field of nonlinear electromagnetic radiation is a source of inflation in the early universe.

The structure of the paper is thus: In section II, we briefly introduced the cosmology of a universe filled with nonlinear magnetic monopole field. In section III, we obtain the evolution of the universe filled with nonlinear magnetic monopole field and variable gravitational constant. In section IV, we checked the stability of the model and give our conclusion.

\section{NON-LINEAR MAGNETIC MONOPOLE FIELDS AND COSMOLOGY}

In nonlinear electrodynamics, we defined the Lagrangian density by [10]

$$
\mathcal{L}_{N E D}=-\frac{\mathcal{F}^{\alpha}}{4}
$$

$\mathcal{F}$ denotes an invariant quantity known as the Maxwell invariant. Since the matter Lagrange is independent of the metric's derivatives, in tensorial language the matter energy-momentum defination using (1) is given as [12]

$$
T_{\mu \nu}=-K_{\mu \lambda} F_{\nu}{ }^{\lambda}+g_{\mu \nu} \mathcal{L}_{N E D},
$$

with

$$
K_{\mu \lambda}=\frac{\partial \mathcal{L}_{N E D}}{\partial \mathcal{F}} F_{\mu \lambda}
$$

Here, it is assumed that on the cosmic Background, there exist a dominant stochastic magnetic field whose wavelengths are less than its curvature. Hence, the mean electromagnetic fields then become the source of Einstein equations [62]. The averaged electromagnetic fields are given by [2]:

$$
\begin{gathered}
\langle E\rangle=\langle B\rangle=0,\left\langle E_{i} B_{j}\right\rangle=0, \\
\left\langle E_{i} E_{j}\right\rangle=\frac{1}{3} E^{2} g_{i j},\left\langle B_{i} B_{j}\right\rangle=\frac{1}{3} B^{2} g_{i j},
\end{gathered}
$$

where \langle\rangle denotes averaging brackets used for taking mean of volume. The wavelength of radiation is considered to be lower than the volume and the volume smaller than the curvature.

However, the case of real nonlinear magnetic monopole is when $E^{2}=0$. Therefore, as obtained from equation (1), the energy density $\rho=-T_{0}^{0}$ and the pressure $p=T_{i}^{i} / 3$ of the nonlinear monopole magnetic field is [5]

$$
\begin{aligned}
& \rho_{N E D}=-\mathcal{L}_{N E D}, \\
& p_{N E D}=\mathcal{L}_{N E D}-\frac{2 B^{2}}{3} \frac{\partial \mathcal{L}_{N E D}}{\partial \mathcal{F}}
\end{aligned}
$$

where $\mathcal{L}_{N E D}$ is defined in Eq. (1) with $\mathcal{F}=B^{2} / 2$.

From the above equations, we obtained the energy density equation $\rho$ and pressure $p$ as thus:

$$
\begin{aligned}
& \rho=\rho_{N E D}=\frac{2^{-\alpha}\left(B^{2}\right)^{\alpha}}{4} \\
& p=p_{N E D}=\frac{2^{-\alpha}}{12}\left(B^{2}\right)^{\alpha}(4 \alpha-3) .
\end{aligned}
$$




\section{COSMOLOGY WITH VARIABLE $G$ AND NONLINEAR ELECTRODYNAMICS}

In varying $G$ theories, the action is still

$$
S=\int d x^{4}\left(\sqrt{-g}\left(\frac{R}{16 \pi G}+\mathcal{L}_{N E D}\right)\right) .
$$

Taking the variation of the action with respect to the metric and ignoring surface terms leads to

$$
G_{\mu \nu}-g_{\mu \nu} \Lambda=\frac{8 \pi G}{c^{4}} T_{\mu \nu}
$$

In a cosmological context, the Friedmann Robertson Walker metric for variable speed of light $c$ and the Newtonian gravitational constant $G$ can be written as

$$
d s^{2}=-c^{2} d t^{2}+a(t)^{2}\left[\frac{d r^{2}}{1-K r^{2}}+r^{2} d \Omega\right],
$$

where $a(t)$ is the scale factor, $t$ the comoving time and $K=0,1,-1$ represent a flat, closed and open FRW universe, respectively.

For the case of flat FRW $(K=0)$ and $c=1$, the Einstein's equations are,

$$
\begin{aligned}
H^{2} & =\left(\frac{\dot{a}}{a}\right)^{2}=\frac{8 \pi G(t)}{3} \rho \\
\frac{\ddot{a}}{a} & =-\frac{4 \pi G(t)}{3}(\rho+3 p) .
\end{aligned}
$$

Where $H$ represents the Hubble parameter and dot is the differentiation with respect to time.

However, the conservation equation that follows from (11)-(12) is for time variation in $G(t)$ is now [42, 43]:

$$
\dot{\rho}+3 \frac{\dot{a}}{a}(\rho+p)=-\rho \frac{\dot{G}}{G} .
$$

the above conservation equation can be written in this forms:

$$
\dot{\rho}+3 \frac{\dot{a}}{a}(\rho+p)+\rho \frac{\dot{G}}{G}=0,
$$

where the equation of state parameter is $\omega=\frac{p}{\rho}$ denotes the equation of state parameter for the dark energy. [52] gives the speed of light $c$ and the gravitational constant $G$ in form of the power-low of the scale factor as:

$$
G=G_{0} a^{m} .
$$

where $G_{0}$ is a positive constant. Since we know that $G$ increases with time, $m$ must be positive.
From the conservation equation (13) we obtain

$$
-\frac{\partial \mathcal{L}_{N E D}}{2 \partial \mathcal{F}} \cdot\left(\frac{\mathrm{d}}{\mathrm{d} t}\left((B(t))^{2}\right)+4 \frac{B(t)^{2} \dot{a}}{a}\right)-\frac{\mathcal{L}_{N E D} \dot{G}}{G}=0 .
$$

The solution of the above equation gives an important relation beteen $B(t)$ and $a(t)$ as.

$$
B(t)=a(t)^{-1 / 2 \frac{4 \alpha+m}{\alpha}} B_{0} .
$$

Conviniently written in terms of the scale factor, the evolution of energy density and pressure is given by:

$$
\begin{gathered}
\rho=\frac{2^{-\alpha}}{4}\left(B_{0}{ }^{2} a(t)^{\frac{-4 \alpha-m}{\alpha}}\right)^{\alpha}, \\
p=\frac{2^{-\alpha}}{12}\left(B_{0}{ }^{2} a(t)^{\frac{-4 \alpha-m}{\alpha}}\right)^{\alpha}(4 \alpha-3) .
\end{gathered}
$$

Then we have

$$
\begin{gathered}
\rho+p=\frac{2^{-\alpha}}{3}\left(B_{0}^{2} a(t)^{\frac{-4 \alpha-m}{\alpha}}\right)^{\alpha} \alpha, \\
\rho+3 p=2^{-1-\alpha}\left(B_{0}{ }^{2} a(t)^{\frac{-4 \alpha-m}{\alpha}}\right)^{\alpha}(2 \alpha-1),
\end{gathered}
$$

and the EoS parameter $\omega$ is

$$
\omega=\frac{4}{3} \alpha-1 . .
$$

Eq. (22) shows some interesting cases of the universe. It follows that at $\alpha=0, \omega=-1$, is a cosmological constant case, at $\alpha=0.5, \omega=-1 / 3$ indicates case of dark energy and at $\alpha=1, \omega=1 / 3$ denotes the case of ultrarelativistic. The matter content of the universe is related to its acceleration via Einstein by:

$$
\frac{\ddot{a}}{a}=-\frac{4 \pi G(t)}{3}(\rho+3 p) .
$$

On checking the singularity in energy density and pressure at $a(t) \rightarrow 0$ and $a(t) \rightarrow \infty$, we obtained,

$$
\begin{gathered}
\lim _{a(t) \rightarrow 0} \rho(t)=\lim _{a(t) \rightarrow 0} p(t)=0, \\
\lim _{a(t) \rightarrow \infty} \rho(t)=\lim _{a(t) \rightarrow \infty} p(t)=0 .
\end{gathered}
$$

Using the Einstein's field equation and energy momentum tensor, the Ricci Scalar $R$ which gives the curvature of spacetime is calculated

$$
R=8 \pi G_{0} a(t)^{m}(\rho-3 p) .
$$

The Ricci tensor squared $R_{\mu \nu} R^{\mu \nu}$ and the Kretschmann 
scalar $R_{\mu \nu \alpha \beta} R^{\mu \nu \alpha \beta}$ are also obtained as

$$
\begin{gathered}
R_{\mu \nu} R^{\mu \nu}=\left(8 \pi G_{0} a(t)^{m}\right)^{2}\left(\rho^{2}+3 p^{2}\right), \\
R_{\mu \nu \alpha \beta} R^{\mu \nu \alpha \beta}=\left(8 \pi G_{0} a(t)^{m}\right)^{2}\left(\frac{5}{3} \rho^{2}+2 \rho p+3 p^{2}\right), \\
\lim _{a(t) \rightarrow 0} R(t)=\lim _{a(t) \rightarrow 0} R_{\mu \nu} R^{\mu \nu}=\lim _{a(t) \rightarrow 0} R_{\mu \nu \alpha \beta} R^{\mu \nu \alpha \beta}=0 .
\end{gathered}
$$

The nature of the scale factor gives the behaviour of the curvature scalar. By taking of the above equations as the universe accelerates at $a(t) \rightarrow 0, \quad$ we obtained no singularities in the curvature scalar, Ricci tensor and the Kretschmann scalar.

\section{THE EVOLUTION OF THE SCALE FACTOR OF THE UNIVERSE}

The first Friedmann equation with variable $G(t)$ for the flat universe is given by

$$
H^{2}=\left(\frac{\dot{a}}{a}\right)^{2}=\frac{8 \pi G(t)}{3} \rho .
$$

When a particle moves in one dimension in a potential $V(a)$, the equation of motion is

$$
\dot{a}^{2}+V(a)=0
$$

The potential function

$$
V(a)=-1 / 32^{1-\alpha} \pi G_{0}(a(t))^{m+2}\left(B_{0}^{2}(a(t))^{\frac{-4 \alpha-m}{\alpha}}\right)^{\alpha}
$$

is negative and possesses a maximum at $a=a_{c}=-C_{1}$.

Using the (15) and (5), it becomes

$$
\frac{-2^{1-\alpha} \pi G_{0} a^{-4 \alpha+2} B_{0}^{2 \alpha}+3 \dot{a}^{2}}{3 a^{2}}=0,
$$

then we find the scale factor $a(t)$ is equal to

$$
\begin{gathered}
a(t) \approx 1 / 2 \sqrt{B_{0}} 2^{3 / 4} 2^{3 / 4 \alpha^{-1}}\left(G_{0} \alpha^{2}\left(C_{2}-t\right)^{2}\right)^{1 / 4 \alpha^{-1}}(34) \\
3^{-1 / 4 \alpha^{-1}} \pi^{1 / 4 \alpha^{-1}} .
\end{gathered}
$$

When $\alpha=1$, we obtained the universe approaches to the matter dominated model [64]. In the early universe, there is a de Sitter phase because of the nonlinear corrections to Maxwell's theory, which is seen in Fig.1 for $\alpha<1$, especially at $\alpha=0.3$. Thus, the model describes inflation at the early epoch as shown in Fig. 1. Moreover, for $\alpha>1$, the universe enters into its accelerating expansion phase with matter dominated.

Introducing the quantity $q$ (the deceleration parame-

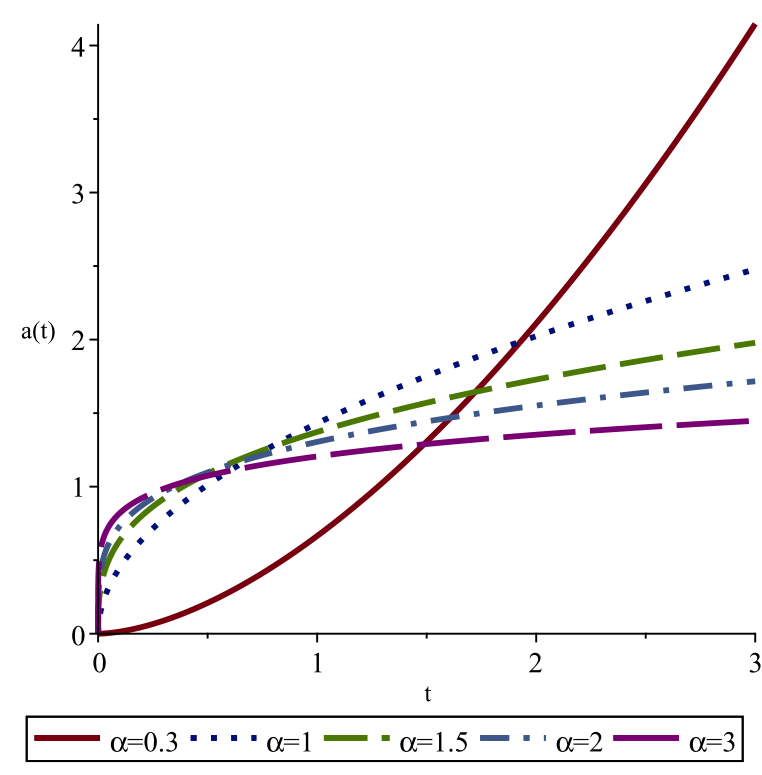

FIG. 1: Plot of the scale factor $a$ versus the time $t$ (for $B_{0}=G_{0}=C_{2}=m=1$ ).

ter) [64], we described the expansion of the universe by:

$$
q=-\frac{\ddot{a} a}{(\dot{a})^{2}}=9 / 2 \frac{\rho+3 p}{a \rho} .
$$

There is a inflation phase for $q<0$ for $\alpha<1$ and deceleration phase for $q>0$ for $\alpha>1$ shown in Fig.2.

To estimate the amount of the inflation, we use the definition of e-foldings

$$
N=\ln \frac{a\left(t_{\text {end }}\right)}{a\left(t_{i n}\right)}
$$

where $t_{\text {end }}$ is the time inflation ends while $t_{i n}$ is the time it begins. For $N \simeq 70$ e-folding, the cosmic flatness and horizon problems can be resolved. Hence, we obtained the scale factor for beginning time of inflation (for $m=$ $\left.1, \alpha=1, G_{0}=1, B_{0}=1\right)$ :

$$
a\left(t_{i n}\right)=3.46 x 10^{-31}
$$

Using the second Friedmann equation Eq. (23) which is known as the acceleration equation for the universe.

$$
\frac{\ddot{a}}{a}=\frac{(1-2 \alpha)}{4 \alpha^{2} t^{2}} .
$$




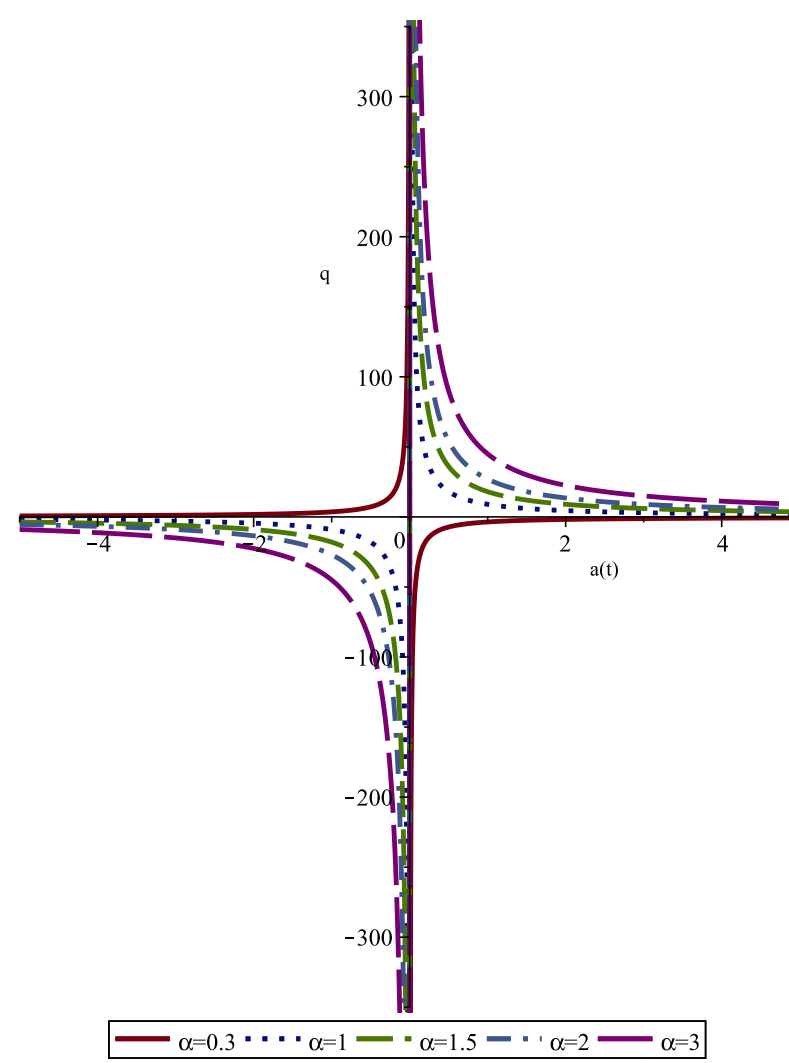

FIG. 2: Plot of the deceleration parameter $q$ versus the scale factor $a$ (for $B_{0}=G_{0}=C_{2}=m=1$ ).

It is clear that acceleration stops at $\alpha=0.5$.

\section{CONCLUSION}

For any cosmological model to survive, it is an established fact that the speed of sound do not exit the local speed of light, $c_{s} \leq 1$. The second veracity that ensures stability requires that the square of the speed of sound is positive, i.e $c_{s}^{2}>0$. In case, the model is a classically stable one [63]. At $E=0$, we obtained:

$$
c_{s}^{2}=\frac{d p}{d \rho}=\frac{d p / d \mathcal{F}}{d \rho / d \mathcal{F}}=-\frac{7}{3}+\frac{4}{3} \alpha .
$$

A requirement of the classical stability $c_{s}^{2}>0$ is $\alpha>\frac{7}{4}$ and the causality $c_{s} \leq 1$ is $\alpha \leq \frac{5}{2}$. Hence, the best value of $\alpha$ for both stability conditions are $\frac{5}{2} \geq \alpha>\frac{7}{4}$.

In this work we have studied cosmology with varying gravitational constant $G$ and Nonlinear Electrodynamics in a flat FRW universe. Under change of scale factor, the evolution of magnetic field reduced to $B(t)=$ $a(t)^{-1 / 2} B_{0}$ as obtained in [12] when $m=0$. The evolution of the scale factor shows that the models gives an accelerating expanding universe with

$$
\begin{array}{r}
a(t) \approx 1 / 2 \sqrt{B_{0}} 2^{3 / 4} 2^{3 / 4 \alpha^{-1}}\left(G_{0} \alpha^{2}\left(C_{2}-t\right)^{2}\right)^{1 / 4 \alpha^{-1}} \\
3^{-1 / 4 \alpha^{-1}} \pi^{1 / 4 \alpha^{-1}}(40)
\end{array}
$$

, where $B_{0}$ represents the magnetic induction field at present time $t_{0}$ and $\beta$ a free parameter presented in Fig. 1 . When $\alpha=1$ we obtained that the universe becomes the matter dominated universe. In the early universe, it is possible to the de Sitter phase for $\alpha<1$ seen in Fig.1, especially at $\alpha=0.3$. Thus, the model describes inflation at the early epoch as shown in Fig. 1. Moreover, for $\alpha>1$, the universe transition to a matter-dominated era at a later time. Also as observed in the equations (24), (25), and (29), we obtained no singularity in the energy density, pressure and curvature terms respectively. Furthermore, we also studied the stability of the this model and observed that that it depends of the constant $\alpha$ and it is classically stable for $\frac{5}{2} \geq \alpha>\frac{7}{4}$. In future study, we intend to investigate the evolution of the universe with both varying $G$ and $c$, in nonlinear electrodynamics.
[1] S. Capoziello and V. Faraoni, Beyond Einstein Gravity: A Survey of Gravitational Theories for Cosmology and Astrophysics (Springer Science+Business Media B.V., New York, 2011).

[2] V. A. De Lorenci, R. Klippert, M. Novello and J. M. Salim, Phys. Rev. D 65, 063501 (2002).

[3] D. N.Vollick, Phys.Rev. D 78, 063524 (2008).

[4] S. I. Kruglov, Phys. Rev. D 92, 123523 (2015).

[5] A. Övgün, Eur. Phys. J. C 77, 105 (2017).

[6] A. Övgün, G. Leon, J. Magana and K. Jusufi, Eur. Phys. J.
C 78, no.6, 462 (2018).

[7] G. Otalora, A. Övgün, J. Saavedra and N. Videla, JCAP 06, 003 (2018).

[8] P. Sarkar, P. K. Das and G. C. Samanta, [arXiv:2005.05568 [hep-ph]].

[9] A. Golovnev, V. Mukhanov and V. Vanchurin, JCAP 0806, 009 (2008)

[10] M. Novello, et al. Phys.Rev. D 69, 127301 (2004).

[11] M. Novello, E. Goulart, J. M. Salim and S. E. Perez Bergliaffa, Class. Quant. Grav. 24, 3021 (2007). 
[12] S. I. Kruglov, Int. J. Mod. Phys. A 32, 1750071 (2017).

[13] S. I. Kruglov, Annals Phys. 353, 299 (2014).

[14] S. I. Kruglov, Int. J. Mod. Phys. D 25, 1640002 (2016).

[15] S. I. Kruglov, Int. J. Mod. Phys. A 31, 1650058 (2016).

[16] S. Kruglov, Eur. Phys. J. Plus 135, no.4, 370 (2020)

[17] M. Sharif and S. Mumtaz, Eur. Phys. J. C 77, 136 (2017).

[18] L. Campanelli, P. Cea, G. L. Fogli and L. Tedesco, Phys. Rev. D 77, 043001 (2008).

[19] Garcia-Salcedo, Ricardo et al. Int.J.Mod.Phys. A15, 43414354 (2000).

[20] F. V. Mukhanov, et al. Phys.Rev.Lett. 68, 1969-1972 (1992).

[21] E. N. Saridakis, M. Tsoukalas, Phys. Rev. D 93, no.12, 124032 (2016).

[22] H. Sheikhahmadi, E.N. Saridakis, A. Aghamohammadi, K. Saaidi, JCAP 10, 021 (2016).

[23] C.S. Camara, et al. Phys.Rev. D 69, 123504 (2004).

[24] R. Durrer, A. Neronov, Astron Astrophys Rev 21, 62 (2013).

[25] K. E. Kunze, Plasma Phys.Control.Fusion 55, 124026 (2013).

[26] H. Azri and D. Demir, Phys. Rev. D 95, no.12, 124007 (2017).

[27] H. Azri and D. Demir, Phys. Rev. D 97, no.4, 044025 (2018).

[28] H. Azri and A. Bounames, Int. J. Mod. Phys. D 26, no.7, 1750060 (2017).

[29] A. Hernandez-Almada, M. A. Garcia-Aspeitia, J. Magana and V. Motta, Phys. Rev. D 101, no.6, 063516 (2020).

[30] M. A. Garcia-Aspeitia, C. Martinez-Robles, A. Hernandez-Almada, J. Magana and V. Motta, Phys. Rev. D 99, no.12, 123525 (2019).

[31] J. A. Vazquez, L. E. Padilla and T. Matos, Revista Mexicana de Física E, 2020 [arXiv:1810.09934 [astro-ph.CO]].

[32] O. Sert, Mod. Phys. Lett. A 35, no.07, 2050037 (2019).

[33] M. Adak, O. Akarsu, T. Dereli and O. Sert, JCAP 11, 026 (2017).

[34] O. Okcu, C. Corda and E. Aydiner, EPL 129, no.5, 50002 (2020).

[35] M. Cruz, F. Izaurieta and S. Lepe, Eur. Phys. J. C 80, no.6, 559 (2020).

[36] M. Cruz and S. Lepe, Class. Quant. Grav. 35, no.15, 155013 (2018).

[37] M. Cruz, N. Cruz and S. Lepe, Phys. Rev. D 96, no.12, 124020 (2017)

[38] R. Cordero, M. Cruz, A. Molgado and E. Rojas, Gen. Rel. Grav. 46, 1761 (2014)

[39] E. Elizalde,et al. Phys.Lett. B 574, 1-7 (2003).

[40] C. Quercellini , M. Bruni, A. Balbi, D. Pietrobon, Phys.Rev. D 78, 063527 (2008).

[41] M. Born and L. Infeld, Proc. Roy. Soc. Lond. A A144, no.852, 425-451 (1934)

[42] J. D. Barrow and J. Magueijo, Phys. Lett. B 443, 104 (1998).

[43] A. Albrecht and J. Magueijo, Phys. Rev. D 59, 043516 (1999).

[44] A. G. Cohen, D. B. Kaplan and A. E. Nelson, Phys. Rev. Lett. 82, 4971 (1999)

[45] J. Moffat, Eur. Phys. J. C 76, no.3, 130 (2016)

[46] K. Leszczynska, Universe 3, no.2, 46 (2017).

[47] C. Camara, R. Nascimento, J. Carvalho and M. De Garcia Maia, Int. J. Mod. Phys. D 16, 433-437 (2007).

[48] L. Kantha, Adv. Astron. 2016, 9743970 (2016)

[49] S. Nesseris, L. Perivolaropoulos, Phys.Rev. D 73103511 (2006).

[50] H. Weyl, Natturwissenschaften 22, 145149 (1934).

[51] P. A. M. Dirac, Proc. R. Soc. A 165, 199208 (1938).
[52] A. K. Singha and U. Debnath, Int. J. Mod. Phys. D 16, 117122 (2007)

[53] M. S. Singh and S. S. Singh, Turk J Phys 42: 198-209 (2018).

[54] R. Dungan and H. B. Prosper, [arXiv:0909.5416 [astroph.CO]].

[55] M. Malekjani, J. Lu, N. Nazari-Pooya, L. Xu, D. Mohammad-Zadeh Jassur and M. Honari-Jafarpour, Astrophys. Space Sci. 360, 24 (2015).

[56] A. Sheykhi, Phys. Scripta 85, 045901 (2012).

[57] J. Lu, L. Xu, H. Tan and S. Gao, Phys. Rev. D 89, 063526 (2014).

[58] M. Sharif and A. Jawad, Eur. Phys. J. C 72, 1901 (2012).

[59] J. Lu, E. N. Saridakis, M. R. Setare and L. Xu, JCAP 1003, 031 (2010).

[60] A. Sheykhi and M. R. Setare, Int. J. Theor. Phys. 49, 2777 (2010).

[61] B. Borah and M. Ansari, Int. J. Theor. Phys. 53, 1217 (2014).

[62] R. Tolman, P. Ehrenfest, Phys.Rev. 36, no.12, 1791 (1930).

[63] R. Garcia-Salcedo, T. Gonzalez, I. Quiros, Phys.Rev. D 89, no.8, 084047 (2014).

[64] S. M. Carroll, [arXiv:gr-qc/9712019 [gr-qc]]. 